\title{
Exponential Dichotomies and Fredholm Operators of Dynamic Equations on Time Scales
}

\author{
Le Huy Tien, Le Duc Nhien \\ Department of Mathematics, Mechanics and Informatics, Vietnam National University at Hanoi, Hanoi, Vietnam \\ Email: lehuytien78@gmail.com,nhien0610@gmail.com
}

How to cite this paper: Tien, L.H. and Nhien, L.D. (2019) Exponential Dichotomies and Fredholm Operators of Dynamic Equations on Time Scales. Applied Mathematics, 10, 39-50.

https://doi.org/10.4236/am.2019.101004

Received: December 28, 2018

Accepted: January 28, 2019

Published: January 31, 2019

Copyright (๑) 2019 by author(s) and Scientific Research Publishing Inc. This work is licensed under the Creative Commons Attribution International License (CC BY 4.0).

http://creativecommons.org/licenses/by/4.0/

\section{c) (i) Open Access}

\begin{abstract}
For time-varying non-regressive linear dynamic equations on a time scale with bounded graininess, we introduce the concept of the associative operator with linear systems on time scales. The purpose of this research is the characterizations of the exponential dichotomy obtained in terms of Fredholm property of that associative operator. Particularly, we use Perron's method, which was generalized on time scales by J. Zhang, M. Fan, H. Zhu in [1], to show that if the associative operator is semi-Fredholm then the corresponding linear nonautonomous equation has an exponential dichotomy on both $\mathbb{T}^{+}$and $\mathbb{T}^{-}$. Moreover, we also give the converse result that the linear systems have an exponential dichotomy on both $\mathbb{T}^{+}$and $\mathbb{T}^{-}$then the associative operator is Fredholm on $\mathbb{T}$.
\end{abstract}

\section{Keywords}

Exponential Dichotomy, Fredholm Operator, Time Scales, Linear Dynamic Equation

\section{Introduction and Preliminaries}

Exponential dichotomy is at the heart of the fundamental perturbation results for linear systems of Coppel (see [2] [3]) and Palmer (see [4] [5] [6] [7] [8]), of the spectral theory of Sacker and Sell [9] [10], of the geometric theory of Fenichel [11], of perturbation results for invariant manifolds [12], of the fundamental perturbation results for connecting orbits of Beyn and Sandstede (see [13] [14] [15]), and it has proven also a formidable ally to justify and gain insight into the behavior of various algorithmic approaches for solving boundary value problems, for approximating invariant surfaces and for computing traveling waves, among 
other uses (see [16] [17] [18]). Hence, it is important to find the conditions for dynamical systems are exponential dichotomy. In 1988, K. J. Palmer presented Fredholm operator concept to show conditions of systems which have exponential dichotomy (see [4]). Using this concept for nonuniform exponential dichotomies case is presented by L. Barreira, D. Dragicevic and C. Valls (see [19] [20]).

Theory of dynamic equations on time scales was introduced by Stefan Hilger [21] in order to unify and extend results of differential equations, difference equations, $q$-difference equations, etc. There are many works concerned with dichotomies of dynamic equations on time scales (see [22] [23] [24]). The purpose of this paper is to setup and characterize exponential dichotomy in term of Fredholm operators for dynamic equations on time scales.

We now introduce some basic concepts of time scales, which can be found in [25] [26]. A time scale $\mathrm{T}$ is defined as a nonempty closed subset of the real numbers. The forward jump operator $\sigma: \mathbb{T} \rightarrow \mathbb{T}$ is defined by $\sigma(t)=\inf \{s \in \mathbb{T}: s>t\}$ and the graininess function $\mu(t)=\sigma(t)-t$ for any $t \in \mathbb{T}$. In the following discussion, the time scale $\mathbb{T}$ is assumed to be unbounded above and below. We have the following several basis definitions (see [25] [26]).

Definition 1.1. Let $A$ be an $m \times n$ matrix-valued function on $\mathbb{T}$. We say that $A$ is rd-continuous on $\mathbb{T}$ if each entry of $A$ is rd-continuous on $\mathbb{T}$, and the class of all such rd-continuous $m \times n$ matrix-valued funtions on $\mathbb{T}$ is denoted by

$$
C_{r d}=C_{r d}(\mathbb{T})=C_{r d}\left(\mathbb{T}, \mathbb{R}^{m \times n}\right) .
$$

We say that $A$ is differentiable on $\mathbb{T}$ provided each entry of $A$ is differentiable on $\mathbb{T}$, and in this case we put

$$
A^{\Delta}=\left(a_{i, j}^{\Delta}\right)_{1 \leq i \leq m, 1 \leq j \leq n} \text { where } A=\left(a_{i, j}\right)_{1 \leq i \leq m, 1 \leq j \leq n}
$$

Definition 1.2. (Regressivity). An $n \times n$-matrix-valued function $A$ on a time scale $\mathbb{T}$ is called regressive (with respect to $\mathbb{T}$ ) provided

$$
I+\mu(t) A(t) \text { is invertible for all } t \in \mathbb{T}^{\kappa},
$$

and the class of all such regressive and rd-continuous function is denoted

$$
\mathcal{R}=\mathcal{R}(\mathbb{T})=\mathcal{R}\left(\mathbb{T}, \mathbb{R}^{n \times n}\right) .
$$

Throughout this paper we only consider $A(t) \in \mathcal{R} \cap C_{r d}$.

Definition 1.3. Assume $A$ and $B$ are regressive $n \times n$-matrix-valued functions on $\mathbb{T}$. Then we define $A \oplus B$ by

$$
(A \oplus B)(t)=A(t)+B(t)+\mu(t) A(t) B(t) \text { for all } t \in \mathbb{T}^{\kappa},
$$

and we define $\ominus A$ by

$$
(\ominus A)(t)=-A(t)[I+\mu(t) A(t)]^{-1} \text { for all } t \in \mathbb{T}^{\kappa} .
$$

Remark 1.1. $\left(\mathcal{R}\left(\mathbb{T}, \mathbb{R}^{n \times n}\right), \oplus\right)$ is a group.

Definition 1.4. (Matrix Exponential Function). Let $t_{0} \in \mathbb{T}$ and assume that 
$A \in \mathcal{R}$ is an $n \times n$-matrix-valued function. The unique matrix-value solution of the IVP

$$
Y^{\Delta}=A(t) Y, \quad Y\left(t_{0}\right)=I,
$$

where I denotes as usual the $n \times n$-identity matrix, is called the matrix exponential function (at $\left.t_{0}\right)$, and it is denoted by $e_{A}\left(\cdot, t_{0}\right)$.

We collect some fundamental properties of the exponential function on time scales.

Theorem 1.1. (see [25]). If $A, B \in \mathcal{R}$ are matrix-valued function on $\mathbb{T}$, then

(1) $e_{0}(t, s) \equiv I$ and $e_{A}(t, t) \equiv I$,

(2) $e_{A}(\sigma(t), s)=(I+\mu(t) A(t)) e_{A}(t, s)$,

(3) $e_{A}(t, s)=\left[e_{A}(s, t)\right]^{-1}=\left[e_{\odot A^{*}}(s, t)\right]^{*}$,

(4) $e_{A}(t, s) e_{A}(s, \tau)=e_{A}(t, \tau)$,

(5) $e_{A}(t, s) e_{B}(t, s)=e_{A \oplus B}(t, s)$ if $e_{A}(t, s)$ and $B(t)$ commute.

If $n=1$, one have the equivalent definition of the exponential function on time scales by

$$
e_{p}(t, s)=\exp \left\{\int_{s}^{t} \xi_{\mu(\tau)}(p(\tau)) \Delta \tau\right\} \quad \text { with } \xi_{h}(z)= \begin{cases}z & \text { if } h=0 \\ \log (1+h z) / h & \text { if } h \neq 0\end{cases}
$$

For any $p \in \mathcal{R}(\mathbb{T}, \mathbb{R})$ and $s, t \in \mathbb{T}$, where log is principal logarithm.

Throughout this paper, we assume that the graininess of underlying time scale is bounded on $\mathbb{T}^{+}$, i.e., $G=\sup \mu(t)<\infty$. This assumption is equivalent to the fact that there exist positive numbers $m_{1}, m_{2}$ such that for every $t \in \mathbb{T}^{+}$, there exists $c=c(t) \in \mathbb{T}^{+}$satisfying $m_{1} \leq c-t<m_{2}$ (also see ([27], pp. 319)). We refer [25] [26] for more information on analysis on time scales.

Next, we define several concepts functional analysis which is useful later. The operator $T: X \rightarrow Y$ (where $X, Y$ are Banach space), we define

- $N(T)$ is nullspace of $T$ and $\operatorname{nul} T=\operatorname{dim}(N(T))$,

- $R(T)$ is range of $T$ and $\operatorname{def} T=\operatorname{codim}(R(T))$ in $Y$,

- $\operatorname{Ind} T=\operatorname{nul} T-\operatorname{def} T$ (if at least one of them is finite).

Definition 1.5. Let $T \in \mathcal{L}(X, Y)$. We say that $T$ is Fredholm operator if

(1) $R(T)$ is closed,

(2) $\mathrm{Nul} T$ and $\operatorname{def} T$ are finite.

If the condition (2) replace either nulT $<+\infty$ or $\operatorname{def} T<+\infty$ then $T$ is said that semi-Fredholm.

In this paper, we only consider the time scales satisfy $\sup \mathbb{T}=+\infty$ and $\inf \mathbb{T}=-\infty$. We also denote $\mathbb{T}^{+}=[0,+\infty) \cap \mathbb{T}, \mathbb{T}^{-}=(-\infty, 0] \cap \mathbb{T}$.

Definition 1.6. The equation

$$
x^{\Delta}=A(t) x
$$

is said to have an exponential dichotomy or to be exponentially dichotomous on $J\left(J=\mathbb{T}^{+}, \mathbb{T}^{-}\right.$or $\left.\mathbb{T}\right)$ if there exist projections matrix $\{P(t)\}_{t \in J}$ on $\mathbb{R}^{n}$ such that $e_{A}(t, s) P(s)=P(t) e_{A}(t, s)$ for any $t \geq s \geq t_{0}$ and

$\left.e_{A}(t, s)\right|_{\operatorname{Ker} P(s)}: \operatorname{Ker} P(s) \rightarrow \operatorname{Ker} P(s)$ is an isomorphism for any $t \geq s, t, s \in J$ and there exist a positive constants $K_{i}$ and $\alpha_{i}, i=1,2$, such that 
(1) $\left|e_{A}(t, s) x\right| \leq K_{1} e_{\ominus \alpha_{1}}(t, s)|x|$ for all $x \in \operatorname{Range} P(s)$ and any $t \geq s, t, s \in J$,

(2) $\left|e_{A}(t, s) y\right| \leq K_{2} e_{\ominus \alpha_{2}}(s, t)|y|$ for all $y \in \operatorname{Ker} P(s)$ and any $t \leq s, t, s \in J$.

where $t, s \in \mathbb{T}$ and $e_{A}(t, s)$ is fundamental solution matrix of Equation (1) and $I$ is the identity matrix. When previous inequality hold with $\alpha_{1}=\alpha_{2}=0$. is said to possess an ordinary dichotomy. The definition of exponential dichotomy can be seen in [1] [22] [24].

We denote several Banach spaces which shall be used later.

- $B C(J)=\left\{x: J \rightarrow \mathbb{R}^{n} \mid x\right.$ is bounded and $\mathrm{rd}$ - continuous $\}$ with the norm

- $\|x\|=\sup |x(t)|$.

- $B C_{0}(\stackrel{t \in J}{J})=\{x \in B C(J) \mid x$ has compact support in $J\}$.

- $B C^{1}(J)=\left\{x \in B C(J) \mid x^{\Delta}\right.$ is bounded and $\mathrm{rd}$ - continuous $\}$ with the norm

- $\|x\|=\sup _{t \in J}|x(t)|+\sup _{t \in J^{K}}\left|x^{\Delta}(t)\right|$.

- $C_{0}(J)=\{x \in B C(J) \mid x(t) \rightarrow 0$ when $|t| \rightarrow+\infty\}$.

- $L^{p}(J)=\left\{x: J \rightarrow \mathbb{R}^{n} \mid x\right.$ is a Bochner measurable function on $\left.J\right\}$ with the norm

$$
\|x\|_{p}=\left(\int\|x(t)\|_{J}^{p} \Delta t\right)^{1 / p} .
$$

where $p>0$ and $J=\mathbb{T}^{+}, \mathbb{T}^{-}$or $\mathbb{T}$.

Remark 1.2. $C_{0}(J)$ is a closed subspace of $B C(J)$ in which $B C_{0}(J)$ is dense.

With the system (1) we define the bounded associative linear operator $L: B C^{1}(J) \rightarrow B C(J)$ as following

$$
L(x)=x^{\Delta}-A(t) x .
$$

Remark 1.3. Null $L$ is always finite. Hence the assumption that $L$ is semi-Fredholm means that the range $R(L)$ of $L$ is closed.

Follow [24], we say the pair $\left(B C(J), L^{p}(J)\right)$ is admissible for (1) if for every $f \in L^{p}(J)$ there exists a function $x \in B C(J)$ such that the pair $(x, f)$ satisfies

$$
x(t)=e_{A}\left(t, t_{0}\right) x\left(t_{0}\right)+\int_{t_{0}}^{t} e_{A}(t, \tau) f(\tau) \Delta \tau, \quad t>t_{0}, t, t_{0} \in J .
$$

We say that $L^{p}(J)$ is the input space and $B C(J)$ is the output space.

The main aim of this paper is to show that the nonautonomous equations have exponential dichotomy on time scales if and only if its associative operator is Fredholm. We now give an outline of the contents of this paper. In Section 2, we use Perron's method, which was generalized on time scales by J. Zhang, M. Fan, H. Zhu in [1], to show that if the associative operator is semi-Fredholm then the corresponding linear nonautonomous equation has an exponential dichotomy on both $\mathbb{T}^{+}$and $\mathbb{T}^{-}$. As a consequence, we obtain that Fredholm property implies the admissibility of the pair $\left(B C\left(\mathbb{T}^{+}\right) ; L^{p}\left(\mathbb{T}^{+}\right)\right)$. In Section 3, we give the converse of the main theorem of section 2 on the lines. Particularly, 
the system (1) has an exponential dichotomy on both $\mathbb{T}^{+}$and $\mathbb{T}^{-}$then the associative operator $L$ is Fredholm on $\mathbb{T}$.

\section{The Sufficient for Exponential Dichotomy on Both Two Half Lines}

Firstly, we need prove two lemmas that are very useful for the main theorem in this section.

Lemma 2.1. Let $A(t)$ be an $n \times n$ matrix-value function, bounded, rd-continuous and regressive on an interval $J$, when $J=\mathbb{T}^{+}, \mathbb{T}^{-}, \mathbb{T}$. Let $f \in B C_{0}(J)$ then the following statements are satisfy

(1) If $J$ is a half line then there exist $x \in B C_{0}^{1}(J)$ such that $L(x)=f$,

(2) If $J=\mathbb{T}$ then there exist $x \in B C_{0}^{1}(J)$ such that $L(x)=f$ if and only if

$$
\int_{-\infty}^{+\infty} \xi^{*} e_{\ominus A^{*}}^{*}(\sigma(\tau), 0) f(\tau) \Delta \tau, \quad \forall \xi \in \mathbb{R}^{n}
$$

Proof. (1) Let $J=\mathbb{T}^{+}$. Then the solution of the nonhomogenneous equation

$$
x^{\Delta}=A(t) x+f(t)
$$

can be written as

$$
x(t)=e_{A}(t, 0) \xi+\int_{0}^{t} e_{A}(t, \sigma(\tau)) f(\tau) \Delta \tau \quad\left(t \in \mathbb{T}^{+}\right)
$$

Since $f$ has compact support, so there exist $r \geq 0$ such that $f(t)=0$ for all $t \geq r$ and $t \in \mathbb{T}^{+}$. Then, for $t \geq r$, we obtain

$$
\begin{gathered}
x(t)=e_{A}(t, 0) \xi+\int_{0}^{+\infty} e_{A}(t, \sigma(\tau)) f(\tau) \Delta \tau \\
\Leftrightarrow x(t)=e_{A}(t, 0)\left\{\xi+\int_{0}^{+\infty} e_{A}(0, \sigma(\tau)) f(\tau) \Delta \tau\right\}
\end{gathered}
$$

so $x(t)$ has compact support on $\mathbb{T}^{+}$if and only if $\xi=-\int_{0}^{+\infty} e_{A}(0, \sigma(\tau)) f(\tau) \Delta \tau$.

This proves the lemma for $J=\mathbb{T}^{+}$. The proof for $J=\mathbb{T}^{-}$is similar.

(2) Let $J=\mathbb{T}$ then (3) is a solution of (2) for all $t \in \mathbb{T}$. Therefore, $x$ has compact support on $\mathbb{T}$ if and only if $x$ has compact support on both $\mathbb{T}^{+}$and $\mathbb{T}^{-}$. It means that

$$
\xi=-\int_{0}^{+\infty} e_{A}(0, \sigma(\tau)) f(\tau) \Delta \tau=-\int_{0}^{-\infty} e_{A}(0, \sigma(\tau)) f(\tau) \Delta \tau .
$$

Hence,

$$
\int_{-\infty}^{+\infty} e_{A}(0, \sigma(\tau)) f(\tau) \Delta \tau=0
$$

or

$$
\int_{-\infty}^{+\infty} \xi^{*} e_{\ominus A^{*}}^{*}(\sigma(\tau), 0) f(\tau) \Delta \tau=0 \quad \text { for all } \xi \in \mathbb{R}^{n} .
$$


This completes the proof of the lemma.

We now consider $\mathcal{X}=L^{-1}\left(C_{0}(J)\right)$. Since $L$ is continuous and $C_{0}(J)$ is closed in $B C(J)$ so $\mathcal{X}$ is also a closed subspace. Then we define $T: \mathcal{X} \rightarrow C_{0}(J)$ to be the restriction of $L$ to $\mathcal{X}$ and we have $R(T)=C_{0}(J) \cap R(L)$. In the following lemma, we characterize $N\left(T^{*}\right)$, where $T^{*}: C_{0}^{*}(J) \rightarrow \mathcal{X}^{*}$ is the conjugate operator.

Lemma 2.2. Let $A(t), J, T$ are defined as before. Then

(1) when $J=\mathbb{T}^{+}$or $\mathbb{T}^{-}$then $N\left(T^{*}\right)=\{0\}$,

(2) when $J=\mathbb{T}$ then $\alpha \in N\left(T^{*}\right)$ if and only if there exist $\xi \in E^{n}$ such that

$$
\int_{-\infty}^{+\infty}\left|e_{\ominus A^{*}}(\sigma(\tau), 0) \xi\right| \Delta \tau<+\infty \text { and } \alpha(f)=\int_{-\infty}^{+\infty} \xi^{*} e_{\ominus A^{*}}^{*}(\sigma(\tau), 0) f(\tau) \Delta \tau .
$$

Proof. (1) First, let $J=\mathbb{T}^{+}$and consider $f \in B C_{0}(J)$. By Lemma 2.1, the Equation (1) with this $f$ has a solution $x \in B C_{0}^{1}(J)$. Obviously, $x \in \mathcal{X}$ and $f=T x$, i.e., $f \in R(T)$. Therefore, for any $\alpha \in N\left(T^{*}\right)$,

$$
\alpha(f)=\alpha(T x)=\left(T^{*} \alpha\right)(x)=0, \quad \forall f \in B C_{0}(J) .
$$

Note that $B C_{0}(J)$ is dense in $C_{0}(J)$. By the continuity of $\alpha$, we see that $\alpha(f)=0$ for all $f \in C_{0}(J)$. Thus, as a linear functional on $C_{0}(J), \alpha$ must be zero and $N\left(T^{*}\right)=\{0\}$. A similar discussion can be given in the case of $J=\mathbb{T}^{-}$.

(2) We now consider $J=\mathbb{T}$ and take $\alpha \in N\left(T^{*}\right)$ and $f \in B C_{0}(J)$. Let

$$
\tilde{f}(t)=f(t)-\phi(t) \int_{-\infty}^{+\infty} e_{A}(\sigma(t), \sigma(\tau)) f(\tau) \Delta \tau
$$

where $\phi$ is a certainly chose function of compact support with $\phi(t) \geq 0$ and $\int_{-\infty}^{+\infty} \phi(t) \mathrm{d} t=1$.

${ }^{-\infty}$ Clearly, $\tilde{f}$ has compact support and

$$
\begin{aligned}
& \int_{-\infty}^{+\infty} e_{\ominus A^{*}}^{*}(\sigma(\tau), 0) \tilde{f}(\tau) \Delta \tau \\
& =\int_{-\infty}^{+\infty} e_{\ominus A^{*}}^{*}(\sigma(\tau), 0) f(\tau) \Delta \tau-\int_{-\infty}^{+\infty} \phi(\tau) \int_{-\infty}^{+\infty} e_{A}(0, \sigma(\tau)) e_{A}(\sigma(\tau), \sigma(s)) f(s) \Delta s \Delta \tau \\
& =0
\end{aligned}
$$

Thus, $\tilde{f} \in R(T)$. By Lemma 2.1, it implies $\tilde{f}=T x$ with $x \in B C_{0}^{1}(J)$. Since $\alpha \in N\left(T^{*}\right)$ so

$$
\alpha(\tilde{f})=\alpha(T x)=\left(T^{*} \alpha\right)(x)=0
$$

From the formula (5) and direct computations, we obtain

$$
\alpha(f)=\int_{-\infty}^{+\infty} \xi^{*} e_{\ominus A^{*}}^{*}(\sigma(\tau), 0) f(\tau) \Delta \tau
$$

For all functions $f \in B C_{0}(J)$,

$$
|\alpha(f)|=\left|\int_{-\infty}^{+\infty} \xi^{*} e_{\ominus A^{*}}^{*}(\sigma(\tau), 0) f(\tau) \Delta \tau\right| \leq\|\alpha\|\|f\| .
$$


It follows that $\int_{-\infty}^{+\infty}\left|e_{\ominus} A^{*}(\sigma(\tau), 0) \xi\right| \Delta \tau \leq\|\alpha\|<\infty$. Then $\alpha$ and $f \rightarrow \int_{-\infty}^{+\infty} \xi^{*} e^{*} A^{*}(\sigma(\tau), 0) f(\tau) \Delta \tau$ are both bounded linear functionals defined on $B C(J)$ and coinciding on the dense subset consisting of the functions of compact support. So (4) holds for all $f \in B C(J)$, as required.

Conversely, suppose there exist $\xi \in \mathbb{R}^{n}$ such that (4) is true. Then

$$
\begin{aligned}
\int_{-\infty}^{+\infty}\left|\left(e_{\ominus A^{*}}(\tau, 0)\right)\right| \Delta \tau & =\int_{-\infty}^{+\infty}\left|A^{*}(t) e_{\ominus A^{*}}(\sigma(\tau), 0) \xi\right| \Delta \tau \\
& \leq \sup _{t \in \mathbb{T}}\left|A^{*}(t)\right| \int_{-\infty}^{+\infty}\left|e_{\ominus A^{*}}(\sigma(\tau), 0) \xi\right| \Delta \tau<+\infty
\end{aligned}
$$

so that $\int_{0}^{t}\left(e_{\ominus A^{*}}(\tau, 0) \xi\right)^{\Delta} \Delta \tau$ has limits as $|t| \rightarrow+\infty$, hence $e_{\ominus A^{*}}(\tau, 0) \xi$ is also. On the other hand,

$$
\begin{gathered}
e_{\ominus A^{*}}(t, 0) \xi=\left[I+\mu(t) A^{*}(t)\right] e_{\ominus A^{*}}(\sigma(t), 0) \xi \\
\Leftrightarrow\left|e_{\ominus A^{*}}(t, 0) \xi\right| \leq(1+\chi M)\left|e_{\ominus A^{*}}(\sigma(t), 0) \xi\right| \\
\Leftrightarrow \int_{-\infty}^{+\infty}\left|e_{\ominus A^{*}}(\tau, 0) \xi\right| \leq(1+\chi M) \int_{-\infty}^{+\infty}\left|e_{\ominus A^{*}}(\sigma(\tau), 0) \xi\right|<\infty .
\end{gathered}
$$

Therefore, $\lim _{\mid t \rightarrow \infty} e_{\ominus A^{*}}(t, 0) \xi=0$.

Now $\alpha$ defined by (4) is certainly in $C_{0}^{*}(J)$. Moreover, if $x \in \mathcal{X}$ we have

$\left(T^{*} \alpha\right)(x)=\alpha(T x)=\int_{-\infty}^{+\infty} \xi^{*} e_{\ominus A^{*}}^{*}(\sigma(\tau), 0)\left(x^{\Delta}(\tau)-A(\tau) x(\tau)\right) \Delta \tau$

$=\left.\left(e_{\ominus A^{*}}(t, 0) \xi\right)^{*} x(t)\right|_{-\infty} ^{+\infty}-\int_{-\infty}^{+\infty}\left\{\left[e_{\ominus A^{*}}(\tau, 0) \xi\right]^{* \Delta}+\left[e_{\ominus A^{*}}(\sigma(\tau), 0) \xi\right]^{*} A(\tau)\right\} x(\tau) \Delta \tau$

$=\int_{-\infty}^{+\infty}\left\{\left[e_{\ominus A^{*}}(\tau, 0) \xi\right]^{\Delta}+A^{*}(\tau) e_{\ominus A^{*}}(\sigma(\tau), 0) \xi\right\}^{*} x(\tau) \Delta \tau=0$

It means $\alpha \in N\left(T^{*}\right)$ so the proof is complete.

We now prove the main theorem of this section.

Theorem 2.1. Let the system (1) with $A(t)$ is $r d$-continuous, bounded and regressive on time scales $\mathbb{T}$. Suppose that the associative operator $L$ of (1) is semi-Fredholm. Then

(1) When $J=\mathbb{T}^{+}$or $\mathbb{T}^{-}$then (1) has exponential dichotomy on J,

(2) When $J=\mathbb{T}$ then (1) has exponential dichotomy on both $\mathbb{T}^{+}, \mathbb{T}^{-}$.

Proof. Since $R(L)$, the range of the semi-Fredholm operator, is closed. Hence, $R(T)$ is also. Then by Theorem 4.6-C in Taylor [28],

$$
\{R(T)\}^{0}:=\left\{\alpha \in C_{0}^{*}(J): \alpha(f)=0, \forall f \in(T)=N\left(T^{*}\right)\right\}
$$

(1) Suppose now $J=\mathbb{T}^{+}$. Then by Lemma 2.2, $N\left(T^{*}\right)=\{0\}$. So by the Hahn-Banach theorem, $R(T)=C_{0}(J)$. That is, for all $f \in B C_{0}(J)$ then the equation (2) has a solution bounded on $J$. Then it follows from Theorem 3.6 in [1] that equation (1) has an exponential dichotomy on $\mathbb{T}^{+}$. In case $J=\mathbb{T}^{-}$is 
similar.

(2) We now consider $J=\mathbb{T}$. By Lemma 2.2 then nul $T^{*}<+\infty$. Furthermore, $\{R(T)\}^{0}=N\left(T^{*}\right)$. It follows that

$$
R(T)={ }^{0}\left\{N\left(T^{*}\right)\right\}=\left\{f \in C_{0}(J): \alpha(f)=0, \forall \alpha \in N\left(T^{*}\right)\right\}
$$

so $f \in R(T) \Leftrightarrow \alpha(f)=0, \forall \alpha \in N\left(T^{*}\right)$. By Lemma 2.2 again,

$$
f \in R(T) \Leftrightarrow \int_{-\infty}^{+\infty} \xi^{*} e_{\ominus A^{*}}^{*}(\sigma(\tau), 0) f(\tau) \Delta \tau=0
$$

for some $\xi \in E^{n}$ satisfies $\int^{+\infty}\left|e_{\odot A^{*}}(\sigma(\tau), 0) \xi\right| \Delta \tau<+\infty$.

Let any $f \in C_{0}\left(\mathbb{T}^{+}\right)$we åre going to extend the function $f$ as following

Let $\phi_{1}, \phi_{2}, \cdots, \phi_{m}$ be a basis for subspace

$$
V:=\left\{e_{\odot A^{*}}(\sigma(\tau), 0) \xi: \int_{-\infty}^{+\infty}\left|e_{\odot A^{*}}(\sigma(\tau), 0) \xi\right| \Delta \tau<+\infty\right\} .
$$

We now choose a function $g \in C_{0}\left(\mathbb{T}^{-}\right)$such that

- $\int_{-\infty}^{0} \phi_{i}^{*}(\tau) g(\tau) \Delta \tau=-\int_{0}^{+\infty} \phi_{i}^{*}(\tau) f(\tau) \Delta \tau,(i=1, \cdots, m)$

- $g(0)=f(0)$

We define

$$
\tilde{f}(t)= \begin{cases}f(t) & \text { for } t \geq 0 \\ g(t) & \text { for } t<0\end{cases}
$$

Hence, $\tilde{f} \in C_{0}(\mathbb{T})$ and $\int^{+\infty} \phi_{i}^{*}(\tau) \tilde{f}(\tau) \Delta \tau=0$ when $i=1, \cdots, m$. It means that the equation $L x=\tilde{f}$ has solution on $B C(\mathbb{T})$ of the equation

$$
x^{\Delta}=A(t) x+\tilde{f}(t)
$$

has bounded solution on $\mathbb{T}$. Restricting to $\mathbb{T}^{+}$we conclude that equation

$$
x^{\Delta}=A(t) x+f(t)
$$

has bounded solution for all $f \in C_{0}\left(\mathbb{T}^{+}\right)$. By the results in [22] (Theorem 3.6) used earlier, it follows that Equation (1) has exponential dichotomy on $\mathbb{T}^{+}$. A similar argument shows that it has an exponential dichotomy on $\mathbb{T}^{-}$. So the proof of the theorem is complete.

By Theorem 3.1 in [24], one has the following corollary about relation between semi-Fredholm property and admissibility.

Corollary 2.1. If the associative operator of (1) is semi-Fredholm operator and $J=\mathbb{T}^{+}$then pair $\left(B C(J), L^{p}(J)\right)$ is admissible for (1).

With the results above, we showed that if the associative operator is semi-Fredholm then the corresponding linear nonautonomous equation has an exponential dichotomy on both $\mathbb{T}^{+}$and $\mathbb{T}^{-}$. As a consequence, we obtain that Fredholm property implies the admissibility of the pair $\left(B C\left(\mathbb{T}^{+}\right) ; L^{p}\left(\mathbb{T}^{+}\right)\right)$.

\section{The Sufficient for Fredholm Property on the Line}

In this section, we assume that the Equation (1) has exponential dichotomy on 
both $\mathbb{T}^{+}$and $\mathbb{T}^{-}$. Then there exist two projections $P$ and $Q$ that satisfy Definition 1.6. Then the adjoint equation

$$
x^{\Delta}=-A^{*}(t) x^{\sigma}
$$

has exponential dichotomy on $\mathbb{T}^{+}$and $\mathbb{T}^{-}$with the corresponding propositions $I-P^{*}$ and $I-Q^{*}$. Now the subspace of initial values (at $t=0$ ) of bounded solutions of (1) is

$$
E=\left\{\xi: \sup _{t \in J}\left|e_{A}(t, 0) \xi\right|<+\infty\right\}=R(P) \cap N(Q)
$$

and for (6) is

$$
F=\left\{\xi: \sup _{t \in J}\left|e_{\odot A^{*}}(\sigma(\tau), 0) \xi\right|<+\infty\right\}=\left\{\xi: \sup _{t \in J}\left|e_{\odot A^{*}}(t, 0) \xi\right|<+\infty\right\} .
$$

Theorem 3.1. Let $A(t)$ be an $n \times n$ matrix function bounded, $r d$-continuous and regressive on $\mathbb{T}$ such that the system (1) has an exponential dichotomy on both $\mathbb{T}^{+}$and $\mathbb{T}^{-}$. Then

(1) $f \in R(L)$ if and only if

$$
\int_{-\infty}^{+\infty} \xi^{*} e_{\odot A^{*}}^{*}(\sigma(\tau), 0) f(\tau) \Delta \tau=0, \quad \forall \xi \in F,
$$

(2) The associative operator $L$ is Fredholm on $\mathbb{T}$.

Proof. Proof of the part (ii) is similar to Palmer [2]. For the part (1), let $f \in R(L)$ so that there exists $x$ in $B C(\mathbb{T})$ such that

$$
L x=f=x^{\Delta}-A(t) x .
$$

Then if $\xi \in F$ we obtained

$$
\begin{aligned}
& \int_{-\infty}^{+\infty} \xi^{*} e_{\ominus A^{*}}^{*}(\sigma(\tau), 0)\left(x^{\Delta}(\tau)-A(\tau) x(\tau)\right) \Delta \tau \\
& =\left.\xi^{*} e_{\ominus A^{*}}^{*}(\sigma(\tau), 0) x(t)\right|_{-\infty} ^{+\infty}-\int_{-\infty}^{+\infty}\left\{\left[e_{\ominus A^{*}}(\tau, 0) \xi\right]^{* \Delta}+\xi^{*} e_{\ominus A^{*}}^{*}(\sigma(\tau), 0) A(\tau)\right\} x(\tau) \Delta \tau \\
& =0
\end{aligned}
$$

Conversely, suppose $f \in B C^{1}(\mathbb{T})$ and satisfy

$$
\int_{-\infty}^{+\infty} \xi^{*} e_{\ominus A^{*}}^{*}(\sigma(\tau), 0) f(\tau) \Delta \tau=0, \quad \forall \xi \in F
$$

Note that if $\eta$ is a vector satisfying

$$
\eta^{*}[P-(I-Q)]=0,
$$

then the function

$$
\psi(t)= \begin{cases}e_{\ominus A^{*}}(\sigma(t), 0)\left(I-P^{*}\right) \eta & \text { for } t \geq 0 \\ e_{\ominus A^{*}}(\sigma(t), 0) Q^{*} \eta & \text { for } t \leq 0\end{cases}
$$

satisfies (7). It follows that

$$
\eta^{*}\left[\int_{-\infty}^{0} Q e_{\ominus A^{*}}^{*}(\sigma(\tau), 0) f(\tau) \Delta \tau+\int_{0}^{+\infty}(I-P) e_{\ominus A^{*}}^{*}(\sigma(\tau), 0) f(\tau) \Delta \tau\right]=0
$$


for all vectors satisfying (8). This means that the linear algebraic equations

$$
[P-(I-Q)] \xi=\int_{-\infty}^{0} Q e_{\ominus A^{*}}^{*}(\sigma(\tau), 0) f(\tau) \Delta \tau+\int_{0}^{+\infty}(I-P) e_{\ominus A^{*}}^{*}(\sigma(\tau), 0) f(\tau) \Delta \tau
$$

have a solution $\xi$. We consider the function

$$
x(t)= \begin{cases}e_{A}(t, 0) P \xi+\int_{0}^{t} e_{A}(\tau, 0) P e_{\ominus A^{*}}^{*}(\sigma(\tau), 0) f(\tau) \Delta \tau & \text { for } t \geq 0 \\ -\int_{t}^{+\infty} e_{A}(\tau, 0)(I-P) e_{\ominus A^{*}}^{*}(\sigma(\tau), 0) f(\tau) \Delta \tau & \\ e_{A}(t, 0)(I-Q) \xi+\int_{-\infty}^{t} e_{A}(\tau, 0) Q e_{\ominus A^{*}}^{*}(\sigma(\tau), 0) f(\tau) \Delta \tau & \text { for } t \leq 0 \\ -\int_{t}^{0} e_{A}(\tau, 0)(I-Q) e_{\ominus A^{*}}^{*}(\sigma(\tau), 0) f(\tau) \Delta \tau & \end{cases}
$$

is a bounded solution of nonhomogenneous linear system $L x=f$ so that $f \in R(L)$ as required. The Theorem is proved.

As a consequence of the Theorem 3.1, we obtain that the system (1) has an exponential dichotomy on both $\mathbb{T}^{+}$and $\mathbb{T}^{-}$if and only if the associative operator $L$ is Fredholm on $\mathbb{T}$.

\section{Acknowledgements}

The first author was supported in part by the VNU Project of Vietnam National University No. QG101-15.

\section{Conflicts of Interest}

The authors declare no conflicts of interest regarding the publication of this paper.

\section{References}

[1] Zhang, J., Fan, M. and Zhu, H. (2010) Necessary and Sufficient Criteria for the Existence of Exponential Dichotomy on Time Scales. Computers \& Mathematics with Applications, 600, 2387-2398. https://doi.org/10.1016/j.camwa.2010.08.034

[2] Coppel, W.A. (1965) Stability and Asymptotic Behavior of Differential Equations. Heath Mathematical Monographs. Heath \& Co., Boston.

[3] Coppel, W.A. (1978) Dichotomies in Stability Theory. Lecture Notes in Mathematics, Vol. 629, Springer-Verlag, Berlin. https://doi.org/10.1007/BFb0067780

[4] Palmer, K.J. (1988) Exponential Dichotomies and Fredholm. Proceedings of the American Mathematical Society, 104, 149-156. https://doi.org/10.1090/S0002-9939-1988-0958058-1

[5] Palmer, K.J. (1984) Exponential Dichotomies and Transversal Homoclinic Points. Journal of Differential Equations, 55, 225-256. ps://doi.org/10.1016/0022-0396(84)90082-2

[6] Palmer, K.J. (1987) A Perturbation Theorem for Exponential Dichotomies. Proceedings of the Royal Society of Edinburgh Section A, 106, 25-37. https://doi.org/10.1017/S0308210500018175 
[7] Palmer, K.J. (1987) Exponential Dichotomies for Almost Periodic Equations. Proceedings of the American Mathematical Society, 101, 293-298. https://doi.org/10.1090/S0002-9939-1987-0902544-6

[8] Palmer, K.J. (2000) Shadowing in Dynamical Systems: Theory and Applications, Mathematics and Its Applications, Vol. 501, Kluwer Academic Publishers, Dordrecht, Boston.

[9] Sacker, R.J. and Sell, G.R. (1978) A Spectral Theory for Linear Differential Systems. Journal of Differential Equations, 27, 320-358. https://doi.org/10.1016/0022-0396(78)90057-8

[10] Sacker, R.J. and Sell, G.R. (1994) Dichotomies for Linear Evolutionary Equations in Banach Spaces. Journal of Differential Equations, 113, 17-67.

https://doi.org/10.1006/jdeq.1994.1113

[11] Fenichel, N. (1979) Geometric Singular Perturbation Theory for Ordinary Differential Equations. Journal of Differential Equations, 31, 53-98. https://doi.org/10.1016/0022-0396(79)90152-9

[12] Fenichel, N. (1971) Persistence and Smoothness of Invariant Manifolds for Flows. Indiana University Mathematics Journal, 21, 193-226. https://doi.org/10.1512/iumj.1972.21.21017

[13] Beyn, W. (1994) On Well-Posed Problems for Connecting Orbits in Dynamical Systems. Contemporary Mathematics, 172, 131-168.

https://doi.org/10.1090/conm/172/01802

[14] Beyn, W.J. (1990) The Numerical Computation of Connecting Orbits in Dynamical Systems. IMA Journal of Numerical Analysis, 10, 379-405. https://doi.org/10.1093/imanum/10.3.379

[15] Beyn, W.J. and Lorenz, J. (1999) Stability of Traveling Waves: Dichotomies and Eigenvalue Conditions on Finite Intervals. Numerical Functional Analysis and Optimization, 20, 201-244. https://doi.org/10.1080/01630569908816889

[16] Ascher, U., Mattheij, R.M. and Russell, R.D. (1988) Solution of Boundary Value Problems for ODEs. Prentice Hall, Englewood Cliffs.

[17] Broer, H.W., Osinga, H.M. and Vegter, G. (1997) Algorithms for Computing Normally Hyperbolic Invariant Manifolds. Zeitschrift für Angewandte Mathematik und Physik, 48, 480-524. https://doi.org/10.1007/s000330050044

[18] Dieci, L. and Lorenz, J. (1995) Computation of Invariant Tori by the Method of Characteristics. SIAM Journal on Numerical Analysis, 32, 1436-1474. https://doi.org/10.1137/0732066

[19] Barreira, L., Dragicevic, D. and Valls, C. (2016) Fredholm Operators and Nonuniform Exponential Dichotomies. Chaos, Solitons and Fractals, 85, 120-127. https://doi.org/10.1016/j.chaos.2016.01.021

[20] Barreira, L., Dragicevic, D. and Valls, C. (2017) Nonuniform Exponential Dichotomies and Fredholm Operators for Flows. Aequationes Mathematicae, 91, 301-316.

[21] Hilger, S. (1990) Analysis on Measure Chains-A Unified Approach to Continuous and Discrete Calculus. Results in Mathematics, 18, 18-56. https://doi.org/10.1007/BF03323153

[22] Zhang, J., Fan, M. and Zhu, H. (2009) Existence and Roughness of Exponential Dichotomies of Linear Dynamic Equations on Time Scales. Computers \& Mathematics with Applications, 59, 2658-2675. https://doi.org/10.1016/j.camwa.2010.01.035

[23] Zhang, J., Song, Y. and Zhao, Z. (2013) General Exponential Dichotomies on Time Scales and Parameter Dependence of Roughness. Advances in Difference Equations, 
2013, 339 .

[24] Yang, L., Zhang, J., Chang, X. and Liu, Z. (2015) Exponential Dichotomy on Time Scales and Admissibility of the Pair $\left(C_{r d}^{b}\left(\mathbb{T}^{+}, X\right), L^{p}\left(\mathbb{T}^{+}, X\right)\right)$. Advances in Difference Equations, 2015, 69.

[25] Bohner, M. and Peterson, A. (2001) Dynamic Equations on Time Scales: An Introduction with Applications. Birkhauser Boston Inc., Boston. https://doi.org/10.1007/978-1-4612-0201-1

[26] Bohner, M. and Peterson, A. (2003) Advances in Dynamic Equations on Time Scales. Birkhauser Boston Inc., Boston.

[27] Potzsche, C. (2004) Exponential Dichotomies of Linear Dynamic Equations on Measure Chains under Slowly Varying Coefficients. Journal of Mathematical Analysis and Applications, 289, 317-335. https://doi.org/10.1016/j.jmaa.2003.09.063

[28] Taylor, A.E. (1958) Introduction to Functional Analysis. Wiley, New York. 